\title{
ETHICAL ISSUES RELATING TO QUALITATIVE RESEARCH
}

\begin{abstract}
Qualitative research or naturalistic research has moved from the sidelines into the mainstream of health research and an increasing number of qualitative research proposals are being presented for ethical review. Qualitative research presents ethical problems that which are unique to the intensive hands-on paradigm which characterises naturalistic research. This paper briefly outlines the most common methodologies used in this research.

\section{Jelsma J, Clow $\mathbf{S}^{1}$}

${ }^{1}$ School of Health and Rehabilitation Sciences, University of Cape Town.

The four ethical principles of benevolence, non-maleficence, autonomy and justice will be used as a framework to explore specific ethical issues related to this form of inquiry. The need for scientific rigour will also be explored as research that is scientifically unsound can never be ethical.
\end{abstract}

\section{KEY WORDS: QUALITATIVE RESEARCH, ETHICAL PRINCIPLES, INSTITUTIONAL REVIEW BOARD.}

\section{INTRODUCTION}

Whereas qualitative or naturalistic research (DePoy and Gitlin 1994) has formed the cornerstone of research in the social sciences, this approach to scientific inquiry has only recently gained "legitimacy" in the quantitative world of medical science research. Consequently the last decade has witnessed an increase in the number of research proposals presented to review boards and the number of articles published in medical journals that rely on this research approach (Richards and Schwarz 2002). The importance of strictly enforced ethical principles for those who wish to undertake research on human subjects has been recognised for many years. The well-known milestones of the development of the Nuremburg Code of 1949, the Helsinki Declaration (latest version 2000) and subsequent amendments and the publication of the Belmont Report (1979) have all contributed to the growing body of knowledge and the increasing enforcement of the ethical aspect of human research. However, these codes and declarations do not adequately address the issues surrounding qualitative research. This may be for several reasons. Qualitative research was often seen by the "quantitatively driven" medical professions as being "soft research" and consequently very few studies were undertaken using this methodology. It was difficult for a discipline that relied on empirical evidence based on the average responses of as a large number of subjects to accept that useful information could be obtained from samples as small as those used in qualitative research. In addition, qualitative research appeared to be innocuous and capable of far less physical harm to subjects than the medical interventions of surgery or medication.

Medical ethical review boards are now faced with an increasing number of protocols that utilise qualitative research. As these boards may be illequipped to adequately review these protocols this may result in inadequate or inappropriate review of these proposals (Ensign 2003), (Olivier and Fishwick 2003). The majority of ethical board members are unlikely to be aware of the different methodologies that comprise qualitative research which makes review of the scientific merit of the studies very difficult. In addition, although the four principles of, autonomy, justice, non-maleficence and beneficence as outlined by Beauchamp and Childress (Beauchamp and Childress. 2001) are obviously of relevance to qualitative researchers, the specific interpretation of each of these principles by the review boards is not tailored to the peculiarities of qualitative research. There are specific issues, which although relevant to quantitative research are of particular concern to qualitative researchers. These include covert observation, power relationships between researchers and participants, cultural factors, emotional involvement of researchers and participants and private versus public behaviour (Olivier and Fishwick 2003).

This paper will therefore explore how the four principles listed above should be applied to this research paradigm.

\section{SOME DIFFERENCES BETWEEN QUALITATIVE AND QUANTITATIVE RESEARCH PARADIGMS}

For the purposes of this article it is useful to compare quantitative with qualitative research and to highlight the differences between the two. The research questions are very different. The qualitative researcher asks "Why? How?" rather than "How many? How much? How often?" (Hancock 1998). Quantitative research strives for objectivity by posing hypotheses to be accepted or rejected and by asking questions related to the generalisability of the results, i.e. whether it is true for all people within similar categories. Qualitative researchers are more interested in the specific person/s, place or time and are less concerned with how generalisable
CORRESPONDENCE TO:
J Jelsma,
Division of Physiotherapy,
School of Health and Rehabilitation
Sciences, Faculty of Health Sciences, University of Cape Town,
Anzio Road, Observatory 7925 ,
Fax: (27-21) 448-8157
Tel: (27-21) 406-6402.
E-mail: jjelsma@uctgsh1.uct.ac.za 
or transferable the results of the research may be. Qualitative research acknowledges the strength of subjectivity. Quantitative research relies on a deductive approach to the development of theory, i.e. it sets out to prove theories already proposed, whereas qualitative research is based on an inductive approach in that it sets out to develop concepts and theories (Hancock 1998). The deductive approach is intended to verify existing theories, whereas the inductive approach aims to build and develop new theories (DePoy and Gitlin 1994).

The participants in quantitative research often remain anonymous and in order to be able to generalise results, large numbers of participants need to be included. The participants in qualitative research are individuals, each with a voice that is heard by the researcher. They are often chosen, not randomly as in quantitative research but specifically because they have experience or knowledge of the topic under research. In terms of data collection, in qualitative research, the instrument of data gathering is the researcher, not an anonymous questionnaire or set of empirical measurements. (Although anonymous questionnaires may well contain qualitative comments e.g. the Delphi methodology)

The core of qualitative research is the relationship between the researcher and the participants and herein lie many ethical pitfalls. With regard to analysis, quantitative research relies on tests, which if valid and reliable and applied correctly by any statistician will yield exactly the same results. This is not the case in qualitative analysis where the researcher is the key to data analysis and each researcher who approaches the data could interpret the findings somewhat differently. The participants themselves often play an important part in the analysis and interpretation of the findings through peer debriefing and member checking. In participatory action research, these findings are presented to the participants and then form the basis for the next stage of the research or intervention. Qualitative research is often emancipatory in approach.

The presentation of results also differs. Quantitative researchers produce tables and empirical data in which no individual can be individually identified. Qualitative researchers often identify themes or theories that are then supported by extensive reference to the opinions and statements of the individuals who participated in the study. The use of quotations further specifies and individualises the results, although individuals themselves are not identified.

\section{DESIGNS IN QUALITATIVE RESEARCH}

Several different research designs utilised in qualitative research have been identified. These include phenomenology which aims to understand a phenomenon, a happening, a life situation, a social situation, through the use of e.g. case histories in which a person is interviewed and their "lived experience" is shared with the researcher or focus group discussion. Ethnography attempts to "paint a picture" of the community and culture of the participants, whereas grounded theory aims to test the veracity of theories proposed to explain certain phenomena (e.g. the stages of grieving identified by Kubler-Ross) (Hancock 1998). Participatory action research, or endogenous research is another common design in which the research problem and possible methods of resolution of the problem are identified and implemented by the participants (DePoy and Gitlin 1994). These have been well described in the literature and will not be explained here.

\section{PRINCIPLES OF MEDICAL ETHICS APPLIED TO QUALITATIVE RESEARCH \\ Scientific merit:}

Research that is not well designed and which does not have scientific merit can never be ethical as it engages the participants in an enterprise which will not result in useful findings. Both the Nuremberg Code $^{1}$ (Nuremburg Code 1949) and the Declaration of Helsinki (World Medical Association 2000) include statements that good scientific methodology is a prerequisite for ethical justifiability (Dickens 2001). However, if the members of the Institutional Review Board ( IRB) do not understand the principles and design of qualitative research, the IRB is in danger of allowing poorly designed research protocol to proceed. There is a need to ensure representation of members well versed in qualitative research on review boards.

No qualitative proposal should be passed unless the research design is stipulated, the procedures that will be in place to ensure rigour and trustworthiness are described and the methods of data collection and data analysis are clearly stated.

\section{Autonomy}

It is essential to obtain informed consent before any participant is enrolled in a research project. However different research designs present challenges specifically for qualitative researchers in this regard.

The difficulty related to the giving of informed consent to a process which might make changing demands on the participant is a function of emerging research designs. Clearly giving consent to participate in such research cannot be regarded as a once-off procedure but must rather be seen as a process of obtaining and retaining the consent of the participants to remain engaged in the project (Ensign 2003). The issue of participant involvement in the checking of data and interpretation of data also needs to be addressed at the onset. The question of whether the participants have "veto rights" over the final interpretation and presentation of the data also might need to be addressed (Ensign 2003). If participants have final control over the interpretation of the data and reporting thereof, it might introduce a degree of bias. However, the autonomy of the participants might be compromised if this is not granted.

There are also serious issues concerning confidentiality which need to be addressed by the qualitative researcher.

1 The Nuremberg Code: Article 3 states that "The experimentation should be so designed and based on the results of animal experimentation and a knowledge of the natural history of the disease or other problems under study that the anticipated results will justify the performance of the experiment”. Nuremburg Code (1949). Nuremburg Military Tribunals. 
The essence of much qualitative research is that it is specific to a place, a time and often a person. This means that the value of the research resides in the person who provides the information and the researcher who interprets it. It is likely that someone who is familiar with the context in which the research took place will be able to identify the participant and may even be familiar with the events and experiences described. This is true not only of people but, in the case of ethnographic research, communities (either geographical or virtual, such as the community of street children). This may result in stigmatisation of either the individual or the community if controversial information is revealed. The problem of identification of individuals who might have contributed to the data base of his study by others in a small rural community led Fraser to abort his qualitative study on the closure of the local hospital (Fraser 2004). He reported that the study was potentially divisive and could harm the community in which it was to take place.

Ethnographic research, which relies considerably on observation of interactions and processes taking place in a particular setting might require that the researcher engages in covert observation. If the subjects (rather than participants, in this case) are aware of his/her presence it might change their behaviour and invalidate any conclusions that could be drawn. It might even be necessary to deceive the subjects in order to gain trust and obtain confidences (Olivier and Fishwick 2003).

\section{Justice}

The power relationship between researcher and participant is an issue in quantitative research, but far more in qualitative research where the personality and presence of the researcher looms large in the data collection process. Qualitative researchers may wish to investigate the lived experiences of those who are marginalised or different in some way. Such participants may be members of vulnerable populations and the researcher has an obligation to ensure that they have not been exploited or disempowered by the research process (Olivier and Fishwick 2003). Unequal power relationships might make it difficult for the participants to withdraw from the study. It is incumbent upon the researcher to actively flatten the power gradients and ensure that the subjects can freely exercise choice regarding their continued involvement in the study. For example, students might not feel free to express their preferences if interviewed by one of their lecturers.

\section{Non-maleficence:}

Issues of relationships and "leaving the field" (Olivier and Fishwick 2003) or disengaging, feature largely in qualitative research. As mentioned earlier, the investigator is the research instrument and as such needs to establish an atmosphere of trust and openness, particularly with regard to phenomenological research. The intention of the research is to elicit confidences regarding the "lived experience" of the participant. In so doing a relationship is built up in which the most intimate details might be discussed. Sensitive issues may be uncovered, recognised or confronted for the fist time during the research process and the researcher needs to be able to promote the emotional integrity of the participants. The subsequent analysis and presentation of the data might be experienced as "betrayal" by the participant. The possibility of inappropriate dependence on the researcher by the participants or, alternatively too much emotional involvement of the researcher should be recognised in the protocol and strategies for preventing harm should be outlined. This could entail the drawing up of a contract in which the roles of the participant and the researcher are clearly defined. The researcher needs to constantly reflect on their own objectivity and what perspective they might be bringing into the study. Reflexivity refers to the process of self-examination and implies that the qualitative researcher is obliged to continually examine their relationship to the participants and how this might influence the gathering and interpretation of data (DePoy and Gitlin 1994). Regular debriefing sessions with experienced colleagues should also be written into research proposals to assist the researcher to deal with the emotional impact of engaging with abused or disadvantaged participants or communities (Ensign 2003).
Another source of harm might be to the researcher, particularly with regard to ethnographic studies of marginalised communities. Ensign gives the example of observation of street children in which the researchers were often on the streets late at night and exposed to possible physical danger. (Ensign 2003). She suggests that field workers be well prepared in terms of familiarity with the community setting before starting on data collection.

\section{Beneficence}

It can be argued that, whereas this principle is an essential attribute of the research process, the changing of the life situation of the participants is not the goal of research and should be separated from the data gathering role of the researcher (Ensign 2003). However, it is the responsibility of the researcher to ensure that participants are referred to appropriate services whenever the need arises. Occasionally, as in other forms of research, the demands of beneficence can override the principle of autonomy. For example, in the course of qualitative research, particularly with marginalised groups, the researcher might become privy to information regarding the abuse of a child or the desire of a participant to commit a criminal act that would injure others. The responsibility of the researcher to report this information should be made clear to the participants during the informed consent process.

\section{CONCLUSION}

Qualitative research is an essential part of the discovery of new knowledge. It is a very useful complement to quantitative research and there are many studies which now incorporate both a quantitative and qualitative arm. On first sight qualitative research may appear innocuous, however the ethical issues are complex and the danger of emotional harm possibly far greater than that of quantitative research. It is thus incumbent on both researchers wishing to undertake naturalistic studies and IRB who have to review these protocols, to ensure that they are familiar not only with the methodology but also the ethical concerns peculiar to this branch of research. 


\section{REFERENCES}

Beauchamp, T. and J. Childress. (2001). Principles of biomedical ethics. Oxford, New York, Oxford University Press.

DePoy, E. and L. N. Gitlin (1994). Introduction to research. Multiple strategies for health and human services. St Louis, Baltimore, Boston, Mosby.

Dickens, B. (2001). Can science or ethics compromise each other in human subject research? Toronto, University of Toronto Press.

Ensign, J. (2003). "Ethical issues in qualitative research with homeless youths." Journal of Advanced Nursing 43(1): 43-50.

Fraser, J. (2004). "A case report: Ethics of a proposed qualitative study of hospital closure in an Australian rural community." Family Practice 21(1): 87-91.

Hancock, B. (1998). An Introduction to Qualitative Research. Trent Focus for Research and Development in Primary Health Care. Trent, Trent Focus,: 27.

Nuremburg Code (1949). Nuremburg Military Tribunals.

Olivier, S. and L. Fishwick (2003). Qualitative research in sports sciences; Is the bio-ethics model applicable?, Forum Qualitative Socialforsshung/Forum Qualitative Social Research (e-journal). 2004: 49 paragraphs.

Richards, H. M. and L. Schwarz (2002). "Ethics of qualitative research: are there special issues for health services research?" Family Practice 19(2): 135-139.

World Medical Association (2000). Declaration of Helsinki ethical principles for medical research involving human subjects. Edinburgh, World Medical Association: 4.

\section{CASE STUDY:}

\section{Statement of the problem}

A nurse working in a clinic in a township area notices that the number of young people living on the streets who are admitted with drug overdoses seems to be increasing. Being a young woman with an inquiring mind, she decides to find out whether this is in fact so. She formulates a hypothesis that the number has increased.

She examines records and discovers that the average monthly number of admissions is indeed significantly greater than the average number of admissions the previous year (p.05). She has good objective evidence that her hypothesis is true.

But she still does not know "Why?" So she turns to a different research paradigm - qualitative research.

\section{Research options}

She can observe the behaviour of the community of young people (ethnographic design). In so doing she will get to understand how they obtain their drugs, how often they buy drugs and how often they take drugs. She can supplement this knowledge with interviews. She may have to use covert observation.

Phenomenological research can be used to by eliciting one or more case histories in which a person is interviewed and their "lived experience" is shared with the researcher. Focus group discussion is another way in which information regarding a phenomenon can be gathered.

\section{Methodology:}

She decides to interview the young people to get their opinions regarding the increased use of drugs. However, as buying and taking drugs are not legal, she will have to gain their trust before they will share confidences with her. She decides to spend a few nights at a centre for the homeless, posing as a woman who has lost her job and home and has no place to stay. She hopes to gain the trust of some of the young people and then either do a case study or a focus group. Once she has gained the trust of the young people, she will debrief them as to her real reason for being there and get their informed consent to take part in the study. She plans to hold a series of focus group discussions to investigate why the drug abuse has increased. During the course of these discussions it is likely that she will learn much about the participants, maybe more than the participants realise at the initiation of the study. She also anticipates that she will learn about illegal activity. There is also a concern that the participants might see her as a friend and confidant, rather than as a researcher.

\section{THE 2ND INTERNATIONAL PHYSIOTHERAPY CONGRESS SANDTON CONVENTION CENTRE (25 - 28 MAY 2005) The theme of the congress is "The Gaitway to Recovery". The programme at a glance is as follows:}

\section{Thursday, 26 May 2005:}

Keynote Addresses and Plenary Sessions:

- The Changing Role of Physiotherapy in South A frica - N onceba M bambo

- Human Rights and Ethics - Elisabeth Haase

- Management of Chronic Pain - Helgaard Meyer

Concurrent Sessions:

(invited speaker followed by paper presentations)

- Sports - Mr Michael Mabasa

- Public Health - Ms Monica Schlehmeyer

- N eurology - Dr Paulette van Vliet

Workshops:

- CPRG update - N etcare 911

- Drugs and Sports - Dr Dimitri Constantinou

W hat Does it Really Say - Professor Celie Eales

Treatment of pelvic floor dysfunction - M s Hester van A swegen

\section{Friday, 27 May 2005:}

Plenary Sessions:

- Family Intervention in Chronic Diseases - Professor Aimee Stewart

- Cardiopulmonary - Dr Monika O Isen

Concurrent Sessions \& Panel Discussions:

- N eurology - Use of outcome measures in research

Sports \& Disability - Common injuries in disabled athletes and their prevention
- Cardiopulmonary - Multidisciplinary approach to management of a patient in ICU

Plenary Session:

- Evidence - Based Practice - Dr Ina Diener

Concurrent Workshops:

CPRG - Monica O Isen

N eurology - Paulette van Vliet

OMT - Mariano Rocabado

How to write what we want to say...and to get it published Professor Celie Eales

\section{Saturday, 28 May 2005}

Plenary Session:

Burden of disease and HIV/ Aids - Professor Jenny Jelsma

HIV / AIDS in children - Joanne Potterton

Sports - Professor Tim N oakes

Concurrent Session \& Panel Discussion

- Occupational Health - Physical performance v/ s work demands

Education

Sports - Policy of selection into $\mathrm{N}$ ational Teams - criteria for fitness

Musculoskeletal - Evidence-based physiotherapy practice in management of pain

Other Speakers include:

- Mr A mos Masondo (Mayor of Johannesburg),

- Ms Linda Hunter

- Dr Carina Eksteen

- Professor Coopoo

For further information please refer to page 32. 